Rev. Adm. Saúde (On-line), São Paulo, v. 21, n. 82: e279, jan. - mar. 2021, Epub 16 abr. 2021 http://dx.doi.org/10.23973/ras.82.279

ARTIGO ORIGINAL

\title{
Análise do perfil de pacientes que sobrevivem à sepse
}

Analysis of the profile of patients surviving sepsis

\section{Taise Mateus Fernandes ${ }^{1}$, Rivael Marcelino Ribeiro ${ }^{2}$, Mariana Freitas Comin $^{3}$, Valdemira Santina Dagostin ${ }^{4}$, Luciane Bisognin Ceretta ${ }^{5}$, Mágada Tessmann 6}

1. Enfermeira. Prefeitura Municipal de Criciúma, Criciúma SC

2. Enfermeiro. Universidade do Extremo Sul Catarinense (UNESC), Criciúma SC

3. Enfermeira. Docente do Curso de Enfermagem da UNESC, Criciúma SC

4. Enfermeira. Coordenadora adjunta do Curso de Enfermagem da UNESC, Criciúma SC

5. Enfermeira. Docente do Curso de Enfermagem da UNESC, Criciúma SC

6. Enfermeira. Docente do Curso de Enfermagem da UNESC, Criciúma SC

\section{RESUMO}

Objetivo: Avaliar o perfil dos pacientes sobreviventes à sepse em um hospital privado do sul catarinense. Métodos: Foi realizado uma pesquisa de abordagem quantitativa, transversal, documental, exploratória e de campo, no qual a coleta de dados foi realizada a partir de dados secundários oferecidos pela instituição na qual a pesquisa foi realizada. Resultados: A maioria dos pacientes pesquisados eram mulheres, com sítio infeccioso predominantemente urinários, gastrointestinais e respiratórios, com manifestação da sepse em maio e agosto, com necessidade de reinternação e desenvolvimento de choque séptico. Conclusão: Observou-se que é essencial 
avaliar a qualidade de vida desses pacientes após sua alta hospitalar, para ter certeza de que o tratamento foi adequado e eficiente, reduzindo, consequentemente, os danos originados por essa doença, os quais influenciam diretamente na condição de saúde de cada indivíduo.

Palavras-chave: Sepse, Perfil, Sobreviventes.

\begin{abstract}
Objective: To evaluate the profile of patients surviving sepsis in a private hospital in southern Santa Catarina. Methodology: A quantitative, transversal, documentary, exploratory and field research was carried out, in which data collection was carried out based on secondary data offered by the institution where the research was carried out. Results: Most of the patients surveyed were women, with an infectious site predominantly urinary, gastrointestinal, and respiratory, with sepsis manifestation in May and August, requiring rehospitalization and development of septic shock. Conclusion: It was observed that it is essential to assess the quality of life of these patients after their hospital discharge, to be sure that the treatment was adequate and efficient, consequently reducing the damage caused by this disease, which directly influence the condition of health of everyone.
\end{abstract}

Keywords: Sepsis, Profile, Survivors.

\title{
INTRODUÇÃO
}

Os hospitais são, na atualidade, as instituições sociais responsáveis por garantir a assistência médica, tanto curativa como preventiva, ao alcance da população em geral. As intervenções de saúde que são realizadas podem ser de média e/ou alta complexidade. As unidades de tratamento intensivo (UTI) têm por objetivo o atendimento de pacientes em estado crítico, os quais exigem assistência e observação contínua de médicos e enfermeiros. Ainda, foram criadas com o objetivo de concentrar recursos profissionais e humanos aperfeiçoados para o atendimento a pacientes graves, em estado crítico ${ }^{1}$.

A equipe de enfermagem possui um papel de suma importância na UTI, que vai além do conhecimento técnico inerente à profissão, abarcando também a necessidade de empatia e cuidado para com paciente e familiares. Em um momento delicado como é o período de permanência na UTI, no qual a fragilidade física e emocional se faz presente, é necessário que o profissional de enfermagem possua ainda mais cuidados no exercício da profissão ${ }^{2}$. 
Ainda que a saúde venha apresentado importantes avanços nas últimas décadas, algumas doenças continuam a assolar a população, como é o caso da sepse. Apesar de os números de óbitos devido à sepse terem diminuído nos últimos anos, as sequelas que a doença provoca no organismo, mesmo após a alta do paciente, ainda geram preocupações por parte dos órgãos de saúde pública ${ }^{3,4}$.

A sepse é conceituada como uma resposta inflamatória a uma infecção, que pode ser sistêmica ou localizada e é iniciada pelas endotoxinas liberadas pelo microrganismo infectante. Essas desencadeiam um processo inflamatório sistêmico por meio de mediadores inflamatórios causando alterações microvasculares, lesão endoteliais disseminadas e extravasamento de fluidos, que geram edema intersticial e hipovolemia, afora os distúrbios de coagulação 5. Esses eventos desencadeiam diminuição do aporte de oxigênio e nutrientes para a célula, acometendo consequentemente, as funções de vários órgãos e sistemas ${ }^{6}$.

A sepse se configura em um grave problema de saúde pública em unidade de terapia intensiva e um grande desafio para os profissionais da saúde. No Brasil, a sepse é a segunda principal causa de mortalidade em UTI, com incidência de 82,7 casos em 100.000 habitantes em 1979, de 240,4 por 100.000 em 2004, com mortalidade entre 28 e $60 \%$ de acordo com a gravidade da doença. Com a evolução da sobrevida de pacientes com doenças crônicas e graves, nota-se maior tempo de hospitalização de pessoas portadoras dessas doenças e aumento da incidência de sepse ${ }^{7}$.

Estudos demonstram que o risco de óbito aumenta nos anos seguintes à alta hospitalar, sendo imprescindível que haja um cuidado e acompanhamento do paciente por parte da equipe de enfermagem ${ }^{8,3}$. Ainda, as repercussões geradas no organismo do paciente acometido por sepse permanecem durante anos, e causam diversas disfunções orgânicas, físicas, psicológicas e sociais, afetando diretamente na qualidade de vida ${ }^{3}$.

Portanto, o presente estudo se justifica na relevância atual ocasionada pelo alto número de sobreviventes que apresentam complicações após um episódio de sepse, exigindo assim que haja o conhecimento de como a sepse repercute na qualidade de vida dos pacientes, para que seja possível buscar estratégias que visem à melhoria na sua qualidade de vida pós alta hospitalar. Dessa forma, 0 presente estudo teve como objetivo avaliar o perfil dos pacientes sobreviventes à sepse em um hospital privado do sul catarinense.

\section{METODOLOGIA}

A abordagem metodológica optada para o estudo foi quantitativa, transversal, documental, exploratória e de campo. A pesquisa foi realizada em um hospital privado do sul catarinense. Foram utilizados dados secundários oriundos do banco de dados do referido hospital, que desenvolve atividades relativas à qualidade de vida de pacientes com diagnóstico de sepse. 
Participaram todos os pacientes que apresentaram sepse que sobreviveram do ano de 2019 e constavam no protocolo e banco de dados da sepse do referido hospital. A variável dependente elencada foi a qualidade de vida de pacientes que sobreviveram à sepse. As variáveis independentes estão identificadas como: idade, sexo, grau de escolaridade, estado civil, causa da internação, comorbidades.

Os critérios para inclusão no estudo foi que o participante tivesse sepse durante o período de internação. Os critérios para exclusão no estudo foi não estar no protocolo de sepse e qualidade de vida no período.

Para coleta de dados, foi solicitada a autorização do hospital, para desenvolvimento da pesquisa, sendo oficializada, mediante assinatura da carta de aceite. Posteriormente, o projeto foi encaminhado ao Comitê de Ética em Pesquisa da Universidade do Extremo Sul Catarinense (UNESC) e somente após a aprovação foi dado início ao desenvolvimento do projeto. O número da aprovação do CEP foi 3.889.254.

O módulo WHOQOL-BREF é constituído de 26 perguntas (sendo a pergunta número 1 e 2 sobre a qualidade de vida geral), as respostas seguiram a escala de Likert (de 1 a 5, quanto maior a pontuação melhor a qualidade de vida). Fora essas duas questões ( 1 e 2), o instrumento tem 24 facetas as quais compõem 4 domínios, que são: físico, psicológico, relações sociais e meio ambiente $^{9}$. O hospital adaptou o instrumento relacionado ao WHOQOL-BREF.

\section{RESULTADOS}

Nos resultados obtidos a partir do banco de dados secundário de um hospital privado do extremo sul catarinense, a partir de um total de 100 participantes, quanto ao sexo de pacientes sobreviventes à sepse predominou mulheres com $55 \%$, seguidos do sexo masculino com $43 \%$.

Quando avaliada a faixa etária dos pacientes, $16 \%$ tinham entre 21 e 30 anos e 81 e 90 anos, respectivamente. Entre $61-70$ anos; $71-80$ anos foram $13 \%$; entre 51-60 anos foram $12 \%$; entre $41-50$ anos $7 \%$; $0-10$ anos $4 \% ; 11-21$ anos $3 \%$ e entre $91-94$ anos $2 \%$.

$\mathrm{Na}$ avaliação sobre os convênios que os pacientes possuíam, por se tratar de uma Instituição privada, observou-se, que 67\% tinham Unimed, 18\% SC Saúde e $12 \%$ foi internado como particulares.

Quanto aos meses em que os pacientes sobreviventes da sepse foram hospitalizados, observou-se que o mês de julho predominou com $19 \%$, seguidos de junho com $11 \%$, abril, maio, e outubro com $10 \%$, março, novembro e dezembro com $8 \%$, janeiro com $7 \%$ e agosto com $6 \%$. Quando condensados por período, observou-se que em maio a agosto predominaram com $46 \%$ dos casos, seguidos por janeiro a abril com $27 \%$ e setembro a dezembro com $26 \%$.

Ao se avaliar o foco da infecção que deflagrou a sepse nos pacientes sobreviventes, a situação, observou-se, que predominou infecções do sistema 
geniturinário predominou com $24 \%$, seguidos por sistema gastrointestinal com $23 \%$, sistema respiratório com $22 \%$, sistema tegumentar e infecções sistêmicas com $3 \%$, respectivamente e sistema neurológico $1 \%$, sendo identificada como meningite. Na avaliação da presença de choque séptico em pacientes sobreviventes de sepse, observou-se que $73 \%$ não apresentaram, enquanto $18 \%$ apresentaram.

Na relação sítio de infeção e faixa etária, há significância e os dados demonstram predominância no sistema urinário com faixa etária de 40 anos em $26,1 \%$ dos pacientes, respiratórias com $22,7 \%$ com faixa etária entre 70 e 80 anos respectivamente, gastrointestinal com $17,4 \%$ com idade em torno de 70 anos, cutânea com 33,3\% com faixa etária de 60,70 e 80 anos e sistêmicas com 33,1\%, com faixa etária de 30,50 e 70\%. A Tabela 1 apresenta a tabulação cruzada sobre infecção e faixa etária.

Tabela 1. Infecção x faixa etária e teste de qui-quadrado.

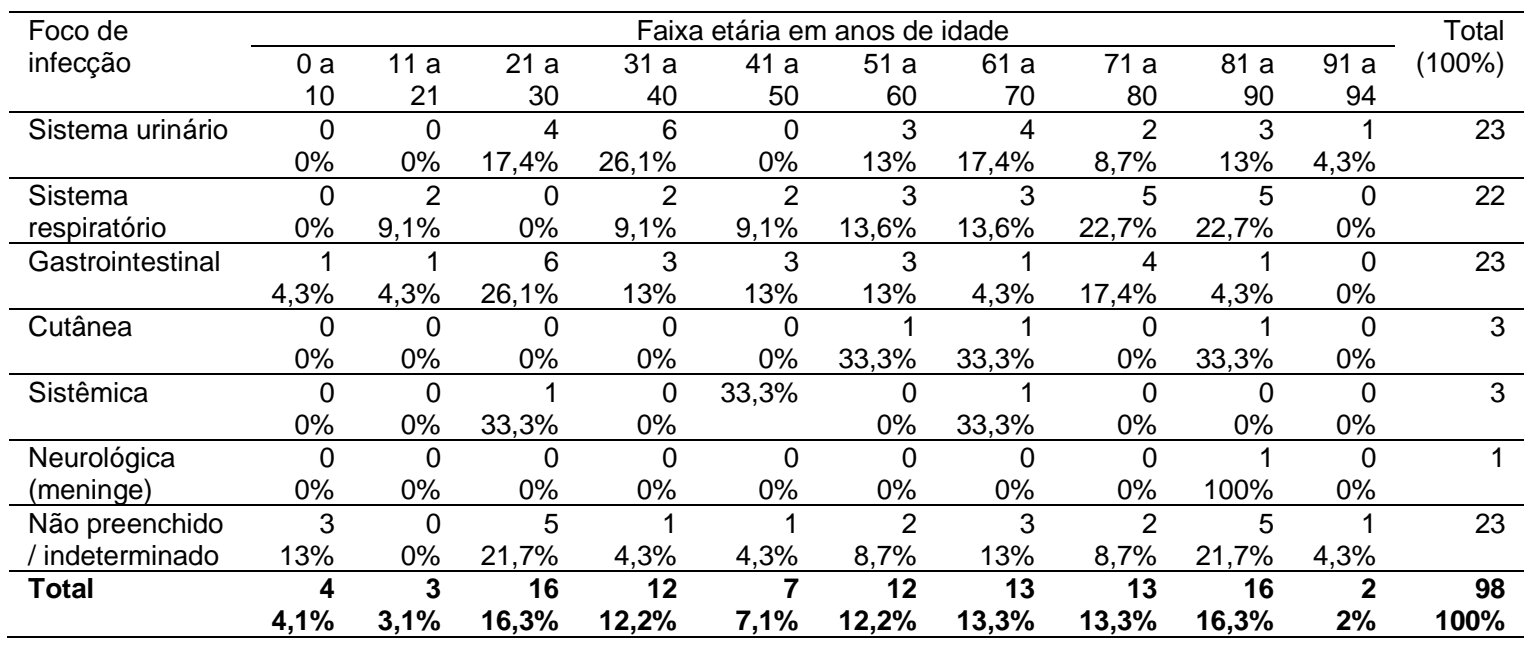

Na Tabela 2 será apresentada o teste de qui-quadrado.

Tabela 2. Teste de qui-quadrado.

\begin{tabular}{lrrr}
\hline & Valor & df & Sig. Assint. (2 lados) \\
\hline Qui-quadrado de Pearson & $49,323^{\mathrm{a}}$ & 54 & 0,655 \\
\hline Razão de verossimilhança & 55,582 & 54 & 0,415 \\
\hline Associação linear por linear & 0,104 & 1 & 0,747 \\
\hline Número de casos válidos & 98 & & \\
\hline a 70 células (100\%) esperam contagem menor do que 5. A contagem mínima esperada é 0,02.
\end{tabular}

Quando avaliada a reinternação hospitalar dos sobreviventes da sepse, observou-se, que 33\% não apresentaram reinternação enquanto $15 \%$ apresentaram. Os registros demostraram que $52 \%$ não dos dados relacionados 
a isso, não estavam preenchidos. Com relação a reinternação e a faixa etária, observa-se significância após aplicação do qui-quadrado, e evidenciou-se que $20 \%$ dos pacientes que apresentaram reinternação hospitalar estavam na faixa etária entre 21 e 30 anos e 51 e 60 anos respectivamente. Foi demonstrado na Tabela 3 uma tabulação cruzada sobre a reinternação e faixa etária.

Tabela 3. Reinternação x faixa etária.

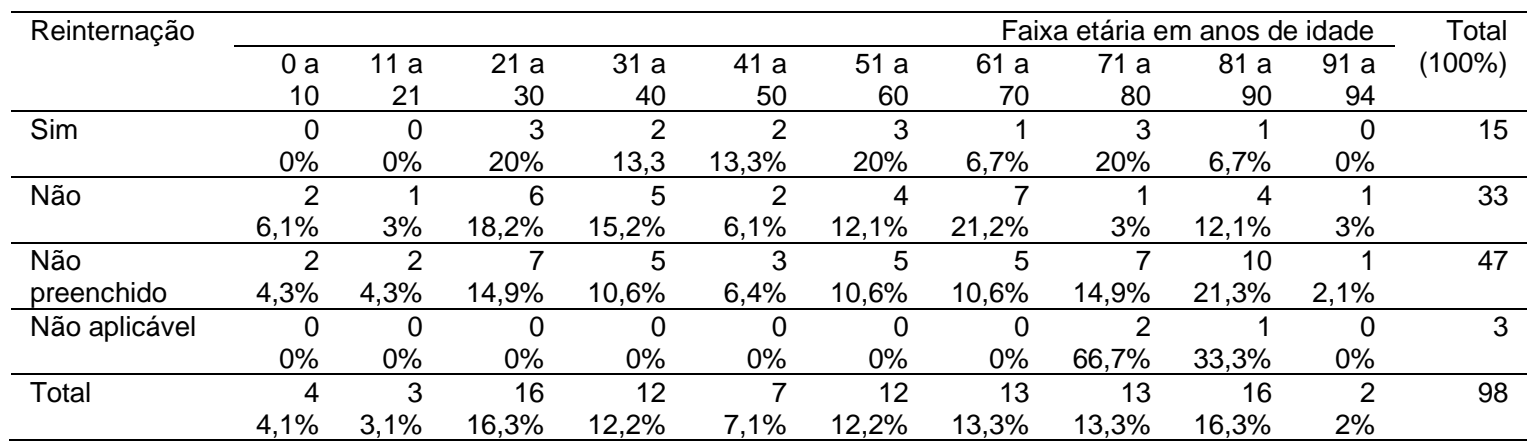

Na Tabela 4 será apresentada o teste de qui-quadrado.

Tabela 4. Teste de qui-quadrado

\begin{tabular}{lrrr}
\hline & Valor & df & Sig. Assint. (2 lados) \\
\hline Qui-quadrado de Pearson & $20,938^{\mathrm{a}}$ & 27 & 0,789 \\
\hline Razão de verossimilhança & 21,955 & 27 & 0,740 \\
\hline Associação linear por linear & 1,920 & 1 & 0,166 \\
\hline Número de casos válidos & 98 & & \\
\hline
\end{tabular}

32 células $(80 \%)$ esperam contagem menor do que 5 . A contagem mínima esperada é 0,06 .

$\mathrm{Na}$ avaliação da relação reinternação e sexo, exposta na Tabela 5, houve significância e os dados revelaram que as mulheres apresentam mais reinternação sendo 66,7\%.

Tabela 5. Reinternação x sexo.

\begin{tabular}{lrrr}
\hline Reinternação & Sexo & & Total \\
\hline & Feminino & Masculino & \\
\hline Sim & 10 & 5 & 15 \\
& $66,7 \%$ & $33,3 \%$ & $100 \%$ \\
\hline Não & 16 & 17 & 33 \\
& $48,5 \%$ & $51,5 \%$ & $100 \%$ \\
\hline Não preenchido & 26 & 21 & 47 \\
& $55,3 \%$ & $44,7 \%$ & $100 \%$ \\
\hline Não aplicável & 3 & 0 & 3 \\
\hline
\end{tabular}




\begin{tabular}{rrrr}
\hline & $100 \%$ & $0 \%$ & $100 \%$ \\
\hline Total & 55 & 43 & 98 \\
& $56,1 \%$ & $43,9 \%$ & $100 \%$ \\
\hline
\end{tabular}

Na Tabela 6 será apresentada o teste de qui-quadrado.

Tabela 6. Teste de qui-quadrado.

\begin{tabular}{lcrr}
\hline & Valor & Df & Sig. Assint. (2 lados) \\
\hline Qui-quadrado de Pearson & $3,817^{a}$ & 3 & 0,282 \\
\hline Razão de verossimilhança & 4,948 & 3 & 0,176 \\
\hline Associação linear por linear & 031 & 1 & 0,861 \\
\hline Número de casos válidos & 98 & & \\
\hline a2 células (25\%) esperam contagem menor do que 5. A contagem mínima esperada é $1,32$.
\end{tabular}

a2 células (25\%) esperam contagem menor do que 5 . A contagem mínima esperada é 1,32.

Foi avaliado o desfecho dos pacientes sobreviventes à sepse, observou-se que $75 \%$ tiveram alta por melhora, $15 \%$ foram a óbito e $6 \%$ foram transferidos. A maioria dos pacientes (75\%) tiveram alta da UTI por melhora, $15 \%$ por óbito e $6 \%$ por transferência. Na avaliação da relação entre desfecho e idade, houve significância e os dados demonstraram que $17,3 \%$ dos que tiveram alta por melhora $28,6 \%$ (4) estavam com faixa etária entre 61-70 anos. A Tabela 7 apresenta a relação desfecho x faixa etária.

Tabela 7. Desfecho x faixa etária.

\begin{tabular}{|c|c|c|c|c|c|c|c|c|c|c|c|}
\hline \multirow{2}{*}{ Desfecho } & \multicolumn{10}{|c|}{ Faixa etária em anos de idade } & \multirow{2}{*}{$\begin{array}{r}\text { Total } \\
(100 \\
\%) \\
\end{array}$} \\
\hline & 0 a 10 & $\begin{array}{r}11 a \\
21 \\
\end{array}$ & $\begin{array}{r}21 a \\
30 \\
\end{array}$ & $\begin{array}{r}31 a \\
40 \\
\end{array}$ & $\begin{array}{r}41 a \\
50 \\
\end{array}$ & $\begin{array}{r}51 a \\
60 \\
\end{array}$ & $\begin{array}{r}61 a \\
70 \\
\end{array}$ & $\begin{array}{r}71 \mathrm{a} \\
80 \\
\end{array}$ & $\begin{array}{r}81 \text { a } \\
90 \\
\end{array}$ & $\begin{array}{r}91 \text { a } \\
94 \\
\end{array}$ & \\
\hline \multirow{2}{*}{$\begin{array}{l}\text { Alta por } \\
\text { melhora }\end{array}$} & 3 & 2 & 13 & 11 & 6 & 10 & 8 & 9 & 12 & 1 & \multirow[t]{2}{*}{75} \\
\hline & $4 \%$ & $2,7 \%$ & $17,3 \%$ & $14,7 \%$ & $8 \%$ & $13,3 \%$ & $10,7 \%$ & $12 \%$ & $16 \%$ & $1,3 \%$ & \\
\hline \multirow{2}{*}{$\begin{array}{l}\text { Não } \\
\text { preenchido }\end{array}$} & 0 & 0 & 2 & 0 & 0 & 0 & 0 & 1 & 0 & 0 & \multirow[t]{2}{*}{33} \\
\hline & $0 \%$ & $0 \%$ & $66,7 \%$ & $0 \%$ & $0 \%$ & $0 \%$ & $0 \%$ & $33,3 \%$ & $0 \%$ & $0 \%$ & \\
\hline \multirow[t]{2}{*}{ Óbito } & 0 & 0 & 1 & 0 & 1 & 1 & 4 & 3 & 3 & 1 & \multirow[t]{2}{*}{47} \\
\hline & $0 \%$ & $0 \%$ & $7,1 \%$ & $0 \%$ & $7,1 \%$ & $7,1 \%$ & $28,6 \%$ & $21,4 \%$ & $21.4 \%$ & $7,1 \%$ & \\
\hline \multirow[t]{2}{*}{ Transferência } & 1 & 1 & 0 & 1 & 0 & 1 & 1 & 0 & 1 & 0 & \multirow[t]{2}{*}{3} \\
\hline & $16,7 \%$ & $16,7 \%$ & $0 \%$ & $16,7 \%$ & $0 \%$ & $16,7 \%$ & $16,7 \%$ & $0 \%$ & $16,7 \%$ & $0 \%$ & \\
\hline Total & $\begin{array}{r}4 \\
4,1 \%\end{array}$ & $\begin{array}{r}3 \\
3,1 \%\end{array}$ & $\begin{array}{r}16 \\
16,3 \%\end{array}$ & $\begin{array}{r}12 \\
12,2 \%\end{array}$ & $\begin{array}{r}7 \\
7,1 \%\end{array}$ & $\begin{array}{r}12 \\
12,2 \%\end{array}$ & $\begin{array}{r}13 \\
13,3 \%\end{array}$ & $\begin{array}{r}13 \\
13,3 \%\end{array}$ & $\begin{array}{r}16 \\
16,3 \%\end{array}$ & $\begin{array}{r}2 \\
2 \%\end{array}$ & 98 \\
\hline
\end{tabular}

Na Tabela 8 será apresentada o teste de qui-quadrado.

Tabela 8. Teste de qui-quadrado.

\begin{tabular}{lccr}
\hline & Valor & Df & Sig. Assint. (2 lados) \\
\hline Qui-quadrado de Pearson & $26,383^{\text {a }}$ & 27 & 0,497 \\
\hline Razão de verossimilhança & 27,113 & 27 & 0,458 \\
\hline Número de casos válidos & 98 & & \\
\hline
\end{tabular}

a33 células (82,5\%) esperam contagem menor do que 5. A contagem mínima esperada é 0,06. 


\section{DISCUSSÃO}

O presente estudo buscou avaliar o perfil dos pacientes sobreviventes à sepse, sendo essa uma resposta inflamatória a uma infecção. Com a realização da mesma observou-se que outros autores também investigaram o perfil em outros locais, como Farias et al. ${ }^{10}$ que realizou um estudo que apresentou perfil dos pacientes com diagnóstico positivo para sepse, que demonstrou que $60,8 \%$ eram do sexo feminino com idade média de 51,5 anos.

A média da faixa etária de paciente com sepse encontra-se na quinta década 11. Outro estudo contrapõe-se e mostra que, em relação ao perfil dos pacientes, a maioria era do sexo masculino $(62,2 \%)$ enquanto $37,75 \%$ era mulheres ${ }^{12}$. 0 presente estudo demonstrou que de um total de 100 pacientes $55 \%$ eram do sexo feminino.

Além disso, Moura et al. ${ }^{12}$ mostraram que a faixa etária onde mais se apresentou sepse, foi de 51 a 70 anos. No estudo de Farias et al. ${ }^{10}$, a idade média dos pacientes variou de 17 a 84 anos. No presente estudo, a idade variou de 0-94 anos, prevalecendo em maior número de 61 a 80 anos.

Batista et al. ${ }^{13}$ mostraram em seu estudo que a distribuição uniforme mensal das internações pode ter como justificativa a condição clínica individual de evoluir ou não para gravidade e haver a necessidade de cuidados intensivos, não associando à sazonalidade, com exceção para algumas doenças como bronquiolite, varicela e dengue nos casos de crianças.

Moura et al. ${ }^{12}$ desenvolveram uma pesquisa que demonstrou em relação ao perfil dos pacientes, $62,25 \%$ são do sexo masculino e $37,75 \%$ do sexo feminino. Os autores mencionam ainda que a taxa de letalidade para sepse continua muito elevada. No Brasil, essa realidade fica ainda mais evidente quando são observados dados de estudos que apontam importante diferença na letalidade da sepse em outros países do mundo. A idade, o sexo masculino, a raça negra e o aumento do fardo das condições crônicas de saúde são fatores de risco importantes para a sepse. O presente estudo demonstrou que $55 \%$ (55) são mulheres, contrapondo-se à pesquisa de Moura et al. ${ }^{12}$.

Barros, Maia e Monteiro ${ }^{7}$ realizaram um estudo que demonstrou que 0 principal foco das infecções de pacientes internados em UTI com sepse foi o pulmonar (43\%), seguida do abdominal (30\%), e as comorbidades mais frequentes foram a diabetes mellitus (19,9\%), a hipertensão arterial sistêmica (HAS) e as neoplasias (16,3\%). No presente estudo, predominaram infecção no sistema urinário, gastrointestinal e respiratório.

Os fatores de risco para sepse grave estão relacionados tanto à predisposição de um paciente para a infecção, quanto à probabilidade de disfunção orgânica aguda, se a infecção se desenvolver. Existem muitos fatores de risco bem conhecidos para as infecções que mais comumente precipitam sepse grave e choque séptico, incluindo doenças crônicas (por exemplo, síndrome da imunodeficiência adquirida, doença pulmonar obstrutiva crônica e muitos 
cânceres) e o uso de agentes imunossupressores. Entre os pacientes com essas infecções, no entanto, os fatores de risco para disfunção orgânica são menos bem estudados, mas provavelmente incluem o organismo causador e a composição genética do paciente, o estado de saúde subjacente e a função preexistente do órgão, além da oportunidade da intervenção terapêutica ${ }^{14}$. 0 presente estudo demonstrou que 18\% (18) apresentaram choque séptico como complicação do quadro de sepse grave.

Juncal et al. ${ }^{15}$ demonstraram, em um estudo, que o tempo prolongado de internação hospitalar dos pacientes sépticos observado nesse estudo (31,9 \pm 30,0 dias), pois isso pode indicar possíveis complicações dos pacientes ao saírem da UTI ou até mesmo a necessidade de reinternação na UTI. No presente estudo $33 \%$ dos pacientes sobreviventes de sepse grave tiveram reinternação hospitalar.

O estudo de Juncal et al. ${ }^{15}$ mostrou ainda que a mortalidade de pacientes sépticos em UTI, assim como a mortalidade hospitalar, foi superior àquela de pacientes não sépticos, evidenciando o impacto dessa síndrome clínica e reforçando a importância do tratamento precoce para minimizar os efeitos sobre os desfechos clínicos. Constatou-se, também, que a mortalidade hospitalar de pacientes sépticos $(60,0 \%)$ foi muito maior do que a encontrada na UTI (38,5\%). Esse fato pode fundamentar uma abordagem mais cautelosa desses pacientes, já que um estudo mostrou que pacientes com quadros prévios de sepse podem ser ameaçados por suas complicações até 5 anos depois, pois o episódio agudo de sepse pode levar a alterações orgânicas irreversíveis que podem repercutir tardiamente, como, por exemplo, a piora de doenças crônicas. Na presente pesquisa, observou-se que 75\% (75) dos pacientes tiveram alta da UTI por melhora.

Westphal et al. ${ }^{16}$ identificaram, em seu estudo, 217 pacientes com sepse grave ou choque séptico, dos quais $112(51,6 \%)$ sobreviveram à internação. Desses, $27(24,1 \%)$ faleceram após a alta e $63(56,3 \%)$ foram confirmados como sobreviventes. A probabilidade acumulada de sobrevida associada à sepse grave e ao choque séptico foi de $41,02 \%$ (80/195) em 6 meses, 37,4\% (73/195) em 12 meses, 34,3\% (67/195) em 18 meses e 32,3\% (63/195) em 24 meses. Apesar deste estudo não identificar correlação entre tipo de alta e faixa etária, pode-se afirmar que houve grande percentual de sobrevida dos pacientes pós sepse e o presente estudo revelou que $17,7 \%$ dos pacientes avaliados que tiveram alta por melhora estava na faixa etária de 21-30 anos.

Zonta et al. ${ }^{17}$ mencionam que vários estudos demonstraram que a rápida identificação da sepse, associada à terapêutica adequada, traz resultados adequados para o paciente. $O$ uso de antimicrobianos específicos na primeira hora, logo após o diagnóstico, contribui significativamente para um desfecho favorável ao paciente. Mas, para que a identificação seja precoce e o tratamento adequado, é fundamental para a aplicação efetiva dos protocolos de sepse e o treinamento da equipe multiprofissional, principalmente da equipe de enfermagem, para que os profissionais estejam aptos a identificar os sinais e as manifestações clínicas da sepse, evitando assim sua complicação. Dos prontuários avaliados, $73 \%$ dos pacientes não apresentaram choque séptico, e 
o hospital no qual foi realizado o estudo tem protocolos específicos, o que pode corroborar com o sugerido por Zonta et al. ${ }^{17}$.

A despeito de a sepse poder estar relacionada a qualquer foco infeccioso, as infecções mais comumente associadas à sua ocorrência são a pneumonia, a infecção intra-abdominal e a infecção urinária. Pneumonia, na maior parte dos levantamentos epidemiológicos, é o foco responsável pela metade dos casos. São ainda focos frequentes a infecção relacionada a cateteres, abcessos de partes moles, meningites, endocardites, entre outros. $O$ foco infeccioso tem íntima relação com a gravidade do processo.

O foco de infecção urinário primário à sepse pode ser justificado pelo fato de que a maioria dos pacientes com sepse grave era mulheres e elas apresentam cistite e outras situações de comorbidades do trato geniturinário com mais frequência.

As infecções respiratórias surgiram com maior frequência em pessoas de 70 a 89 anos e a sepse se apresentou entre os meses de maio e agosto, sendo esses, no sul do país, os mais frios, o que podem ocasionar, sobretudo, maior vulnerabilidade a situações do trato respiratório em idosos.

\section{CONCLUSÃO}

Atualmente, a sepse vem acometendo muitos pacientes, mas, em contrapartida, essa patologia, apesar de amplamente descrita na literatura, é pouco discutida na prática clínica, e, muitas vezes, a causa de morte é atribuída à patologia de base. Dessa maneira, se torna indispensável aumentar a sensibilização sobre a referida patologia, bem como a realização de novos estudos, a fim de capacitar os profissionais para a implantação de protocolos pertinentes ${ }^{17}$.

O presente estudo revelou que a maioria dos pacientes foram mulheres, com sítio infeccioso predominantemente urinários, gastrointestinais e respiratórios, com manifestação da sepse em maio e agosto, com necessidade de reinternação e desenvolvimento de choque séptico. A grande maioria teve desfecho positivo para alta por melhora, contrapondo-se a várias hipóteses préestabelecidas que previam homens, com média de 35 anos tendo a principal causa da sepse a pneumonia (só se confirmou problemas respiratórios em idosos).

A enfermagem tem papel fundamental quando consegue, durante a internação do paciente, identificar de forma precoce sinais e sintomas sugestivos da sepse mediante protocolos, podendo, dessa forma, intervir melhorando sobrevida com qualidade, bem como, evita complicações de desfechos desfavoráveis ao paciente. Para tanto, é necessário que o enfermeiro tenha conhecimento científico profundo, raciocínio clínico e excelente prática profissional no que diz respeito ao uso de tecnologias leve, leve-dura e duras. 
Sugere-se novos estudos direcionados a avaliação da qualidade de vida destes pacientes, depressão em familiares dos pacientes que foram a óbito, perfil dos pacientes sobreviventes relacionando este a terapêutica utilizada e tempo entre internação e diagnostico da sepse, dor no paciente pós sepse, além de estudos que demonstrem o conhecimento dos enfermeiros sobre sepse e seus protocolos.

\section{REFERÊNCIAS}

1. Nascimento ERP, Trentini M. O cuidado de enfermagem na unidade de terapia intensiva (UTI): teoria humanística de Paterson e Zderad. Rev. latinoam. Enferm. 2004;12(2):250-7.

2. Lelis LS, Amaral MS, Oliveira FM. As ações de enfermagem frente à sepse, uma abordagem do paciente crítico: uma revisão da literatura. Revista Científica FacMais. 2018;12(1):60-75.

3. Lago LB. Estado funcional após a alta de UTI em pacientes sobreviventes de sepse. [Dissertação]. Porto Alegre, 2016.

4. ILAS, Instituto Latino-Americano para Estudos da Sepse. Sepse: um problema de saúde pública. Brasília: CFM, 2015.

5. Ferreira B, Ferreira B, Pinto GLS, da Silva JÁ, Amália J, Dias FA, Raimundo LG, de Oliveira LSR, et al. Sepse: uma revisão de literatura. Revista Esfera Acadêmica Saúde. 2017;2(1):6-18.

6. Pereira MC, Pereira MC, da Cunha TMN, Chiavegato L, Lucato JJJ. Qualidade de vida de pacientes sobreviventes a sepse, sepse grave e choque séptico avaliada por meio de duas escalas. Fisioter. Bras. 2014 jul/ago;15(4):311-7.

7. Barros LLS, Maia CSF, Monteiro MC. Fatores de risco associados ao agravamento de sepse em pacientes em Unidade de Terapia Intensiva. Cadernos Saúde Coletiva. 2016;24(4):388-96.

8. Contrin LM, Paschoal VD, Beccaria LM, Cesarino CB, Lobo SMA. Qualidade de vida de sobreviventes de sepse grave após alta hospitalar. Rev. LatinoAm. Enfermagem. 2013 jun;21(3):795-802.

9. Fleck MPA. O instrumento de avaliação de qualidade de vida da Organização Mundial da Saúde (WHOQOL-100): características e perspectivas. Ciência \& Saúde Coletiva. 2000;5(1):33-8.

10. Farias LL, Pinheiro Júnior FML, Braide ASG, Macieira CL, Araújo MVUM, Viana MCC. Perfil clínico e laboratorial de pacientes com sepse, sepse grave e choque séptico admitidos em uma unidade de terapia intensiva. Rev. Saúde Públ. Santa Cat. 2013 jul/set;6(3):50-60. 
11. Menezes LEFJ, de Negreiros LMV, Maciel LBC, Marques TA, Roballo CA, Baffa AM. Perfil epidemiológico e análise da efetividade para prevenção de óbitos de pacientes inseridos em protocolo de sepse. Rev Soc Bras Clin Med. 2019 jun;17(1)25-30.

12. Moura JM, Sanches E, Pereira R, Frutuoso I, Werneck AL, Contrin LM. Diagnóstico de sepse em pacientes após internação em unidade de terapia intensiva. Arq. Ciênc. Saúde. 2017 out;24(3):55-60.

13. Batista NOW, de Rezende Coelho MC, Trugilho SM, Pinasco GC, de Sousa Santos EF, Ramos-Silva V. Perfil clínico-epidemiológico de pacientes internados em unidade de cuidados intensivos pediátricos. J. Hum. Growth Dev. 2015;25(2):187-93.

14. Pires CCM, Corradi CGA, Greco CV, Amorim DR, Duarte FA, Pereira IMF, et al. Importância do tratamento precoce na sepse grave e choque séptico: impacto no prognóstico. Rev Med Minas Gerais. 2011;21(4):102-5.

15. Juncal VR, de Britto Neto LA, Camelier AA, Messeder OHC, Farias AMC. Impacto clínico do diagnóstico de sepse à admissão em UTI de um hospital privado em Salvador, Bahia. J. Bras. Pneumol. 2011 jan/fev;37(1):85-92.

16. Westphal GA, Vieira KD, Orzechowski R, Kaefer KM, Zaclikevis VR, Mastroeni MF. Análise da qualidade de vida após a alta hospitalar em sobreviventes de sepse grave e choque séptico. Revista Panamericana de Salud Pública. 2012 mar;31(6):499-505.

17. Zonta FNS, Velasquez PGA, Velasquez LG, Demetrio LS, Miranda D, Silva MCBD. Características epidemiológicas e clínicas da sepse em um hospital público do Paraná. Rev. epidemiol. controle infecç. 2018;8(3):224-31.

Recebido: 24 de março de 2021. Aceito: 16 de abril de 2021

Correspondência: Valdemira Santina Dagostin. E-mail: vsd@unesc.net

Conflito de Interesses: os autores declararam não haver conflito de interesses. 
(C) This is an Open Access article distributed under the terms of the Creative Commons Attribution License, which permits unrestricted use, distribution, and reproduction in any medium, provided the original work is properly cited 